\title{
Internet of Things: A Review of Functionality, Applications, Architectures and Challenges
}

\author{
*Saniya Zahoor ${ }^{1}$, Shabir A.Sofi ${ }^{2}$ \\ ${ }^{1}$ Post Graduate Department of Computer Science, University of Kashmir, Hazratbal \\ ${ }^{2}$ Department of Information Technology, National Institute of Technology Srinagar \\ ${ }^{1}$ saniyazahoor.ku@gmail.com, ${ }^{2}$ shabir@nitsri.ac.in
}

\begin{abstract}
The Internet of Things (IoT) is an emerging paradigm that embodies the vision of merging smart objects while utilizing the internet as the backbone of the communication system to establish a smart interaction among physical entities in pervasive environments. In IoT, data is generated in realtime and stored in permanent repositories. Additional data in the form of meta-data that describes things adds to the data volume and to manage this data, architecture is required. IoT finds its applicability in a plethora of applications such as transportation, smart city, smart health, smart environment, home entertainment, sports, etc. but there is no universal architecture for all IoT implementations. We have layered architectures and domain-specific architectures for different IoT applications. Besides a large number of architectures for IoT, it faces several potential challenges such as scalability, reliability, heterogeneity, etc. This paper presents an understanding of the Internet of Things in terms of its functionality, layered and domain-specific architectures, and its potential challenges.
\end{abstract}

Keywords: Internet of Things, Wireless Sensor Networks, Sensors, Computation, Domainspecific architecture.

\section{Introduction}

Internet of Things is the dynamic global network infrastructure based on standard communication protocols that aim at the connectedness between the virtual and physical world [1]. IoT finds its applicability in various pervasive applications such as transportation, smart city, smart health, smart environment, home entertainment, sports, logistics, e-education, agriculture, industrial automation, etc [2]. IoT is the dynamic network infrastructure in which the things including physical and virtual entities are identifiable; communicate among themselves, and interacting with the environment. IoT objects or nodes form the basic functional block of the IoT system and perform various activities such as sensing, actuating, controlling, and monitoring the IoT environment. These objects collect the data from other devices, process the data either locally or remotely, exchange the data with other connected devices, etc as shown in figure 1.

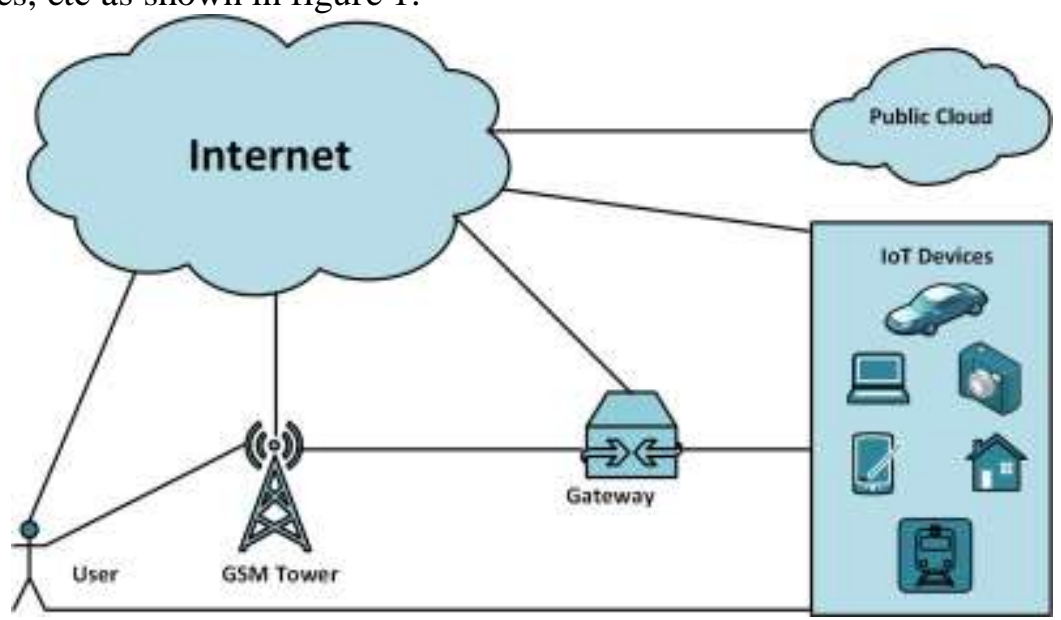

Figure 1. IoT System 
According to Gartner, 25 billion devices will be connected to the internet by 2021 which will generate an enormous amount of data that needs to be analyzed, managed, and make intelligent decisions autonomously. Data generated in real-time and stored in permanent repositories by the IoT objects form the central part of the resources in IoT environments. Additional data in the form of meta-data that describes things adds to the data volume. Thus, IoT data is characterized by many aspects such as massive volume, heterogeneity, and geographical distribution that cause communication and storage overheads. To manage this data, architecture is required. There are different layered architectures and domain-specific architectures for IoT applications [3]. No universal architecture has been proposed to date. Besides a large number of applications, IoT faces several potential challenges such as scalability, availability, mobility, reliability, heterogeneity, etc.

This paper presents an understanding of the Internet of Things in terms of its functionality, applications, layered and domain-specific architectures, and its potential challenges. The organization of the paper is: section 2 presents the functionality of the Internet of Things, section 3 gives various applications of Internet of Things, section 4 discusses the architectures for Internet of Things, section 5 gives the challenges and finally conclusion is given in section 6 .

\section{Functionality of Internet of Things}

Figure 2 summarizes the functionality of Internet of Things. These include sensing, communication, and computation [4].

- Sensing: Gathering the data from IoT devices such as sensors, actuators, etc and sending it to sink that analyze the collected data to take specific actions as per the application requirement.

- Communication: There are several communication protocols such as WiFi, Bluetooth, Zwave, Long-Term Evolution (LTE), etc that connect the various heterogeneous devices in IoT.

- Computation: Computational units and Operating Systems represents the processing ability of an IoT device, hence are called the "brain" of IoT device.

\section{Sensing}

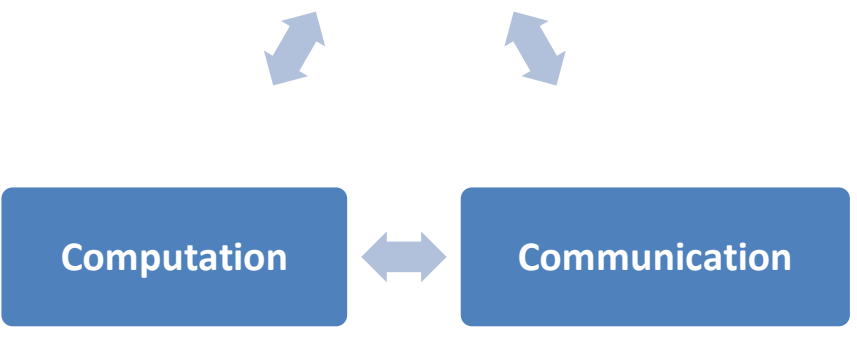

Figure 2. Functionality of Internet of Things

\section{Applications}

Following discusses the various applications of Internet of Things:

\subsection{Emergency}

IoT allows (near) real-time data gathering without human interaction. This is especially important in situations where emergency service team members are busy with critical tasks and additional reporting (e.g. via voice-based radio systems) to the team officer would cause unwanted distraction or delay. For example, smart clothing, equipped with sensors, can report in real time vital signs and temperature of firefighters involved in hazardous situations. A rescue team officer can thus warn 
when the situation gets too hazardous or intervene to rescue the fire fighter in trouble. Such information can be used to alert other team members in real-time in order to act more carefully [5]. When a gas-pipeline that has reached high pressure in a location, and the IoT device informs a remote-control system to regulate the situation to prevent an emergency situation of explosion! This can be seen as a means for utilities to control their networks to prevent emergency situations, through a remote automated closed control system for handling of situations in a faster manner than via human interaction [6]. A critical situation where an IoT device (e.g. industrial control monitor) with a subscription to a priority service needs to send information to a remote server to take action that may include requesting the same or another IoT device to take the action. This IoT device invokes priority service to obtain priority for the data communication session over the communication network. The priority service requires, in addition to the subscription, an application installed on the IoT device responsible to analyze the data received from the sensors and determine when to invoke the priority service. It can also reset itself/revoke the request without human interaction.

\subsection{Smart Transportation}

The automobile industry has concentrated on improving the internal functioning of automobiles [7]. But now, the focus is shifting to improving the in-car experience and making the vehicle more userfriendly. IoT is assisting you in enhancing the experience. There is software that allows you to control your vehicle. These provided you with detailed information on your vehicle. It collects all of your car's data, such as tyre pressure, engine failure, operation and maintenance, fuelling, and so on. Connected automobile solutions are being developed by large automakers as well as some daring start-ups.

\subsection{Industrial Internet of Things}

Industrial Internet, also known as Industrial Internet of Things, is a new buzzword in the industrial sector (IIoT) [8]. Brilliant machines, which are a gift of IoT and are filled with diverse sensors, software, and big data analytics, are operating in this area, making them wiser. These sensors and software collect data, which the Internet of Things interprets to make machines smarter and more accurate and friendly in communicating with humans. The Internet of Things (IoT) aids in realizing the enormous potential for quality control and long-term sustainability.

\subsection{Smart City}

A smart city is another potent IoT application that is garnering interest and excitement among the global population [9]. Surveillance cameras, automated transportation, intelligent energy management systems, water distribution, security, and environmental monitoring are all found in these cities. IoT has the potential to tackle important issues that people experience, such as pollution, traffic congestion, and energy shortages. Many sensors are put in these cities, which are connected to each other and to many other devices via the internet, providing information to users such as parking spaces, any malfunctions, electrical failures, and a variety of other concerns.

\subsection{Smart Wearable}

These are incredibly hot topics in today's society, and demand for them has skyrocketed in marketplaces all around the world. Many businesses are now refocusing their efforts and investing considerably in the development of wearable technology. But how do these contraptions work? These gadgets are equipped with a variety of sensors and software that collect data and information about the users' actions. After then, the data is pre-processed to extract key information about the user. IoT assists in the collection of data and its transmission to manufacturers, allowing them to improve the efficiency and user friendliness of these products [10].

\subsection{Smart Homes}

"Smart Homes" is the most searched word on the internet, causing a lot of confusion and noise. It becomes the cutting-edge ladder of success in the residential Circle, and Smart Homes are expected to become as prevalent as smart phones are now. This technology claimed to save you time, energy, and money, making your life easier and more pleasant than before. Wouldn't it be great if your home followed your thoughts exactly? When you arrive home, they turn on the air conditioning, and when 
you leave, they turn out the lights? Even while you are not at home, you can unlock the doors for friends for temporary access. Don't be surprised; this isn't a dream. IoT is taking shape, and firms are developing solutions to make your life easier and more convenient, and to help you realise your goal. Smart locks, hue bulbs, video doorbells, and wireless lighting are just a few of the devices that may be found in smart homes [11].

\subsection{Weather Monitoring}

Certain metrics, such as humidity, temperature, and pressure in atmosphere etc, must be monitored and controlled in order to track weather conditions [12]. To monitor them, we utilise various sensors to gather data, which we then upload to the cloud, where it will be accessible over the internet from anywhere and we will be able to watch and operate them from afar. Now, cloud-based applications are utilised to access this data, which aids in the early detection of any weather problem by providing alerts, which allow users to be aware of current events and control them from a distance. To monitor the above-mentioned metrics, we have many air and weather monitoring toolkits accessible, such as AirPi, which is highly successful at monitoring these components in the environment and pushing the same data to the internet. There are now a numerous sensors for assessing and tracking condition of weather, such as the DHT 11 sensor in order to check and monitor temperature, the LDR sensor for light detection, the BMP180 sensor for checking and monitoring pressure in air, the raindrops sensor for rainfall, and many others that can be used for various applications.

\subsection{Air Pollution Monitoring}

As we all know, there are many gases in our air that must be in a balanced quantity in the environment. However, owing to air pollution, this does not always occur, necessitating pollution monitoring in order to maintain a healthy and balanced ecosystem.. Large factories in cities or other locations are also responsible for air pollution by emitting toxic or harmful gases that cause pollution and thereby degrading air quality. IoT-based air pollution monitoring allows us to monitor air pollution while also supporting in pollution prevention or reduction. We can make use of smart sensor to to detect changes in gases in air and monitor them as well. The data collected can be pushed on cloud where we can access it. We can also monitor them from afar, allowing us to regulate and monitor gases more effectively. We can even provide real-time analytics and also provide strategic planning right away. To check for threshold levels, there is a chart accessible. Its values should fall within the usual range. If the threshold value is reached or exceeded from normal range, an alert or alarm is generated by some means to help solve the problem. Additionally, an alert can be sent to the entity in charge of environmental preservation and development which will assist the problem right away [13].

\subsection{Forest Fire Detection}

Lightening or immoral acts might cause forest fires. From afar, we are unable to see it. It has an impact on everything from air quality to weather. Using an IoT-based forest fire detection system, we can keep track of this. To check the state of the forest area, we can employ a variety of smart sensors [14]. The sensor detects the environment and captures data such as temperature, humidity, and gases etc in the atmosphere. Data or information can be captured and pushed to the cloud, making it accessible via the internet. We can monitor and control the fire in real time before they spread to avoid any harmful or potentially dangerous situations. We can detect fire by mounting a large number of sensors in locations where fires are more likely to occur. If an issue is noticed, or if any sensor node spots a fire, alert messages can be generated. Multi-hop communication is typically used to cover huge woods. Flame sensors, temperature sensors, and gas sensors etc are all commonly used or some of the most often utilised sensors to detect fire and alert us before any hazardous or dangerous event. Sensor placement is done in a variety of ways. By implanting mobile sensors in animals, we can even detect fire nowadays.

\subsection{Flood Detection}

Floods, flash floods, and other natural disasters are among the world's biggest natural dangers, and they have proven to be a source of concern that must be addressed otherwise lives or property of people are to be lost. IOT has also proven itself in this sector by offering us with an IoT-based smart 
flood monitoring system that helps us avert severe issues with people's lives and helps us to saves their property [15]. IoT assists us in this area by offering improved flood management, allowing us to monitor and take necessary actions to help avert any big issues. We achieve this by placing a range of sensors near flood-prone regions, such as ultrasonic sensors that let us identify water levels, rain sensor to detect situation of rain in that area or velocity sensors that tell us how fast water is flowing right now i.e detects flow rate. Smart sensors actually enable us to collect data and information and push it on the cloud so that we are able to access it through the internet with the help of cloud applications and take necessary actions, such as generating an alert so that if the level of water rises above a certain threshold value or the flow rate rises above a certain range, we can react quickly and handle situation beforehand. Today, we have a variety of novel methods that aid in the early detection of impending floods. We can acquire information via a notification

\subsection{Precision Agriculture}

IoT devices help in increasing the productivity and yield as these not only monitor acid level present in the soil, temperature of soil but helps in monitoring of livestock productivity as well. Even IoT devices are able to provide information to the farmers regarding yield of crops, pest infection, yield of crops, nutrition of soil etc. Hence, IoT devices are capable of providing information to farmers regarding all those variables which in one way or other way affect agricultural yield to a large extent [16].

\section{Architectures}

With sensing, communication, and computational capabilities, the IoT devices, all together form a network, representing several applications. How the IoT devices are deployed forms the basis for the architecture of an application. To date, no universal architecture has been proposed for all IoT implementations. From the survey, we have obtained layered and domain-specific architectures for IoT applications, and these are discussed below [17].

\subsection{Layered IoT architectures}

There are no universal IoT architecture, so different architectures based on the layers present in it has been proposed by different researchers.

- Three-layer Architecture: The three-layer architecture is basic for IoT, consists of three layers i.e; application layer, network layer; and perception layer. Perception Layer (sensor layer) interacts with IoT nodes to measure, collect, process the IoT data, and transmit the processed data into the upper layer. Network Layer (transmission layer) transmits the data from the perception layer to the IoT hub, devices, and applications. Application Layer (Business layer) receives the data transmitted from the network layer. For example, IoT based e-commerce supply chain management is a three-layered architecture [18], M2M-based Municipal involvement architecture has three layered distributed architecture [19], etc.

- Four-layer Architecture: SOA uses the concept of middleware to create an IoT architecture based on the use of system services. Four layers viz., perception layer, network layer, service layer, and application layer exist in SoA-based IoT architecture. The extra layer being service layer aims at service discovery, service composition, service management, and service interfaces. For example, DAVRS aimed at environmental monitoring employs four-layered architecture [20].

- Five-layer Architecture: Five-Layer Architecture consists of five layers viz., perception layer, network layer, processing layer, application layer, and business layer. The additional layers add more functionality such as the processing layer do the computation of data and the business layer manages the business applications e.g., the Home Health Hub Internet of Things (H3IoT) employs five-layered health monitoring architecture [21].

- Six-Layer Architecture: Six layered architecture involve six layers viz., Coding Layer, Perception Layer Network Layer, Processing Layer, Application Layer, Business Layer. The role of the additional layer viz. coding layer is to provide identification to the IoT devices e.g., six-layered WSN agriculture architecture [22]. 


\subsection{Domain-specific IoT Architectures}

Various domain-specific architectures based on the broad application areas are described as under:

- Radio-Frequency Identification (RFID): RFID is the key component of IoT invasion and creates architecture based on things oriented approach. For example, the Electronic Product Code (EPC) in purchase shops [23], Unique identifier (UID) architecture in similar domain IoT applications [24].

- Service-oriented architecture (SOA): SOA uses the concept of middleware to create an IoT architecture based on the use of system services. For example, the INOX platform uses an approach that consists of three layers: Service layer, Platform layer, and Hardware layer [25].

- Wireless Sensor Network (WSN): Wireless Sensor Network is one of the key parts of the IoT system. There are IoT based applications in many domains such as Environment monitoring, Infrastructure Monitoring, Agriculture, Aquaculture, etc. that employ WSN based architecture. For example, Tailings Dam Monitoring and Pre-alarm System (TDMPS) [26], Enose based on aquaculture monitoring architecture [27].

- Supply Chain Management and industry: It is the key IoT component that deals with the flow of goods and services. For example, IoT Mashup-as-a-Service (IoTMaaS) [28], EPC global object service-oriented Resource NameService (RNS) platform [29].

- e-Health care: Smart Healthcare has become possible by various IoT architectures in various areas such as e-Health, m-Health, Ubiquitous health, Hospital management, etc. For example, the Privacy preservation e-Health framework (negotiation based architecture) [30], Ubiquitous Health UDA-IoT architecture [31], Automating Design Methodology (KADS) system for hospital management [32].

- Smart Management: Various IoT architectures are based on the smart society applications that include Road condition monitoring, Traffic management, Smart city, Urban management, etc. For example, Vehicular network using IoT based middleware for traffic monitoring architectures [33], ALMANAC SCP smart city IoT application architecture [34], SENSAPP smart cycling application architecture [35].

- Cloud service and management: Cloud service and management include various architectural solutions for information exchange cloud, Location-aware service, Cognitive service, Control service Sensor discovery service, Fog computing, Big data, etc to encounter cloud computing and big data problems. For example, Fog architecture in HealthCare application [36].

- Social IoT (SIoT): The general SIoT architecture is the same as that of a three-layer IoT architectural model viz., sensing layer, network layer, the application layer. For example, the SIoT Framework for e-Health systems.

\section{Challenges}

The Internet of Things faces several potential open challenges and these are listed below [37].

\subsection{Compatibility}

It becomes challenging to maintain compatibility between different IoT systems when there are so many vendors, OEMs, and service providers. Sensors and networking are essential components of the Internet of Things. However, not every machine has advanced sensors and networking capabilities that allow it to connect and share data effectively. Furthermore, legacy machine sensors with varying power consumption capacities and security standards may not be capable of producing the same findings. Adding external sensors could be a quick fix, but this is difficult since deciding which functions and parts will interact and share data with the network is complicated.

\subsection{Identification and Authentication}

According to a report, there are currently over 20 billion linked devices, and connecting all of them poses a number of security issues in addition to complexity. Bringing a large number of linked devices onto one platform necessitates system design and formalisation that can identify and verify such devices. 


\subsection{Connecting Devices to Platforms}

Enterprises must combine numerous IoT connected goods with the appropriate IoT platforms in order to successfully develop IoT applications. Due to a lack of adequate integration, irregularities in functions and efficiency in delivering value to clients may occur. "Through 2018, half of the cost of establishing IoT solutions will be spent integrating various IoT components with each other and backend systems," says Benoit Lheureux, research vice president at Gartner. It's critical to realise that integration is a critical IoT skill. The main issue here is that there are too many IoT endpoints and assertions that must be connected in order to gather sensor data and send it to an IoT platform. Companies can only mine the massive data using Big Data techniques to produce insight and forecast outcomes if they have deep integration.

\subsection{Connectivity}

It is a part of the networking problems, as the Internet is still not available at the same speed everywhere. According to Inmarsat, a global mobile satellite firm, connection is one of the major hurdles in IoT deployment for $24 \%$ of respondents. Logistics and oil and gas industries that conduct remote operations, in particular, require reliable communication networks to collect data in harsh conditions and communicate it back to the headquarters for analysis. The routers, LAN, MAN, and WAN all play a role in the quality of signals collected by sensors and transmitted to networks. To support rapid and high-quality communication, these networks must be well-connected via various technologies. However, the number of linked devices is rapidly outpacing network coverage, posing monitoring and tracking challenges.

As we all know, all environmental monitoring data is maintained on the cloud, thus having access to the internet is a must. We also have equipment in rural areas; therefore we must ensure that these places have connection to the internet, as data loss can be hazardous to the environment. Connectivity is influenced by a variety of elements, including the device application environment, cost considerations, and the use of outdated technologies. We now have to deal with critical information in a smart environment, such as weather information. Information on floods in some areas, as well as data about fires in fire-prone areas, necessitates precision because these are emergency situations. As a result, poor internet connectivity or a lack of wifi in these locations can lead to trouble and a reason for failure in the system.

\subsection{Unstructured Data}

As the number of connected devices grows, so will the issues of dealing with unstructured data in terms of volume, velocity, and variety. The true challenge for businesses is determining which data is valuable, because only high-quality data is useful. According to a poll, $80 \%$ of today's data is unstructured, which means it can't be saved in SQL format. The unstructured data is kept in NoSQL format, which makes data retrieval a little more difficult. The issue and complexity of processing unstructured data has been decreased with the introduction of Big Data frameworks such as Hadoop and Cassandra, however Big Data is so vast that merging it with IoT poses a significant hurdle. Furthermore, there are no industry-wide standards for data retention and use, as well as metadata.

\subsection{Security and privacy}

One of the primary obstacles or issues faced not only in smart environment monitoring but in every other IOT industry is privacy and security. The security of not only the connected devices, but also the data, is compromised. Also, data in environment monitoring applications in IoT may be exposed to unauthorised access, posing a threat to data privacy. Damage to the environment monitoring infrastructure or a breach of the transmission channel might also jeopardise data confidentiality and integrity. Also, because large environmental data is saved on the cloud, it will inevitably be exposed to the internet, posing a security risk.

\subsection{Energy Consumption}

As we all know, in a smart environment, there are a lot of devices linked, which require a lot of energy and power to keep the network running, thus energy and power consumption is a key issue that needs to be handled. In the smart environment system, we also have a number of sensors that use a lot 
of energy and power. We also have sensors and devices located at distant regions for smart environmental monitoring, thus battery replacement is a concern that can result in some natu some natural disasters if not taken into account. In this case, too small of a battery is a concern.

\subsection{Scalability}

In smart environment monitoring applications, scalability is also a problem. As we all know, a significant number of devices are connected to monitor the environment, and new devices are added to the network on a daily basis. As a result, connecting all devices over a common channel becomes a challenge, as our application must be able to handle this highly scalable environment. While integrating IOT devices in a smart environment, an unstable networking environment is also a big concern. It's also challenging to handle both small and large scale environments.

\section{Conclusions}

Internet of Things is the dynamic global network infrastructure based on standard communication protocols that aim at the connectedness between the virtual and physical world. IoT devices have unique identities and operate in pervasive spaces. Sensing, communication, and computation are fundamental activities of the IoT devices that lead to the generation of real-time data of high volume, and to manage this data, architecture is required. IoT finds its applicability in a plethora of applications but there is no universal architecture for all IoT implementations. There are layered and domain-specific architectures for different IoT applications. Besides a large number of architectures for IoT, it faces several potential challenges such as scalability, reliability, heterogeneity, etc. An understanding of the Internet of Things in terms of its functionality, layered and domain-specific architectures, and its potential challenges are achieved in this paper. The understanding of Internet of Things in these contexts pays a way to research in this field of computing.

\section{REFERENCES}

[1] Zahoor, S. and Mir, R.N., Resource management in pervasive Internet of Things: A survey. Journal of King Saud University-Computer and Information Sciences (2018).

[2] Zahoor, S. and Mir, R.N., IoT fog cloud model for digital reach in rural India. In International Conference on Computer Networks and Communication Technologies (pp. 717-725). Springer, Singapore (2019).

[3] Anderson, J.W., Kennedy, K.E., Ngo, L.B., Luckow, A. and Apon, A.W., October. Synthetic data generation for the internet of things. In 2014 IEEE International Conference on Big Data (Big Data) (pp. 171-176). IEEE (2014).

[4] Ray, P.P., A survey on Internet of Things architectures. Journal of King Saud University-Computer and Information Sciences, 30(3), pp.291-319 (2018).

[5] Sangeetha, SV Tresa, et al. "IoT based Smart Sensing and Alarming System with Autonomous Guiding Robots for Efficient Fire Emergency Evacuation." 2021 2nd International Conference for Emerging Technology (INCET). IEEE, (2021).

[6] Suganya, R., R. Guhan, and N. C. Gowreesan. "Air Quality Monitoring System with Emergency Alerts Using IOT." Journal of Physics: Conference Series. Vol. 1916. No. 1. IOP Publishing, (2021).

[7] Elayyan, Haifaa Omar. "Smart City and Smart Transportation: Intelligent IoT-Based Transportation Objects "MeOnline Mobile Application: A Mutual Practice of Internet of Mobile Things"." Towards Connected and Autonomous Vehicle Highways. Springer, Cham, (2021). 87-128.

[8] Cheng, Jiangfeng, et al. "Industrial IoT in 5G environment towards smart manufacturing." Journal of Industrial Information Integration 10 (2018): 10-19.

[9] Hassan, Rondik J., et al. "State of art survey for iot effects on smart city technology: challenges, opportunities, and solutions." Asian Journal of Research in Computer Science (2021): 32-48.

[10] Kassem, Ahmed, Mohamed Tamazin, and Moustafa H. Aly. "A Context-Aware IoT-Based Smart Wearable Health Monitoring System." International Conference on Communications, Signal Processing, and their Applications (ICCSPA). IEEE, (2021).

[11] Mohammed, M. N., et al. "An internet of things-based smart homes and healthcare monitoring and management system." Journal of Physics: Conference Series. Vol. 1450. No. 1. IOP Publishing, (2020).

[12] Singh, Tanya, and Mohammed Asim. "Weather Monitoring System Using IoT." Innovations in Cyber Physical Systems. Springer, Singapore, (2021).247-253.

[13] Malleswari, S. M. S. D., and T. Krishna Mohana. "Air pollution monitoring system using IoT devices." Materials Today: Proceedings (2021).

[14] Burnade, Dinesh H., et al. "Forest fire detection and prediction using Internet of things."(2021).

[15] Khan, Rijwan, et al. "Early flood detection and rescue using bioinformatic devices, internet of things (IOT) and Android application." World Journal of Engineering (2021).

[16] García, Laura, et al. "IoT-based smart irrigation systems: An overview on the recent trends on sensors and IoT systems for irrigation in precision agriculture." Sensors 20.4 (2020): 1042. 
[17] Malik, S., Dedeoglu, V., Kanhere, S.S. and Jurdak, R., July. TrustChain: Trust management in blockchain and IoT supported supply chains. In 2019 IEEE International Conference on Blockchain (Blockchain) (pp. 184-193). IEEE (2019).

[18] Chen, M., Wan, J. and Li, F., Machine-to-machine communications: Architectures, standards and applications. Ksii transactions on internet \& information systems, 6(2), (2012).

[19] Gu, Q., Liu, Y. and Sun, J., March. DAVRS: an architecture of distributed VR systems. In Fourth International Conference on Virtual Reality and Its Applications in Industry (Vol. 5444, pp. 200-205). International Society for Optics and Photonics (2004).

[20] Ray, P.P., November. Home Health Hub Internet of Things (H 3 IoT): An architectural framework for monitoring health of elderly people. In 2014 International Conference on Science Engineering and Management Research (ICSEMR) (pp. 1-3). IEEE (2014).

[21] Farooq, M.U., Waseem, M., Mazhar, S., Khairi, A. and Kamal, T., A review on internet of things (IoT). International Journal of Computer Applications, 113(1), pp.1-7 (2015).

[22] Brock, D.L., Integrating the Electronic Product Code (EPC) and the Global Trade Item Number (GTIN). White Paper available at www. autoidcenter. org/pdfs/MIT-WUTOID-WH-004. pdf, 25 (2001).

[23] Li, H., Peng, C., Li, B., Chen, Y., Zhang, W., Wu, J. and Huang, H., User ID routing architecture. IEEE Vehicular Technology Magazine, 5(1), pp.62-69 (2010).

[24] Clayman, S. and Galis, A., December. INOX: a managed service platform for inter-connected smart objects. In Proceedings of the workshop on Internet of Things and Service Platforms (pp. 1-8) (2011).

[25] Dong, L., Shu, W., Sun, D., Li, X. and Zhang, L., Pre-alarm system based on real-time monitoring and numerical simulation using internet of things and cloud computing for tailings dam in mines. IEEE Access, 5, pp.21080-21089 (2017).

[26] GholamHosseini, H., Luo, D., Liu, H. and Xu, G., December. Intelligent processing of E-nose information for fish freshness assessment. In 2007 3rd International Conference on Intelligent Sensors, Sensor Networks and Information (pp. 173-177). IEEE (2007).

[27] Im, J., Kim, S. and Kim, D., June. IoT mashup as a service: cloud-based mashup service for the Internet of things. In 2013 IEEE international conference on services computing (pp. 462-469). IEEE (2013).

[28] Pawar, K. and Attar, V., December. A survey on data analytic platforms for Internet of Things. In 2016 International Conference on Computing, Analytics and Security Trends (CAST) (pp. 605-610). IEEE (2016).

[29] Ukil, A., Bandyopadhyay, S., Joseph, J., Banahatti, V. and Lodha, S., August. Negotiation-based privacy preservation scheme in internet of things platform. In Proceedings of the First International Conference on Security of Internet of Things, pp. 75-84 (2012).

[30] Xu, B., Da Xu, L., Cai, H., Xie, C., Hu, J. and Bu, F., Ubiquitous data accessing method in IoT-based information system for emergency medical services. IEEE Transactions on Industrial informatics, 10(2), pp.1578-1586 (2014).

[31] Moreno, L., Aguilar, R.M., Piñeiro, J.D., Estevez, J.I., Sigut, J.F. and Gonzalez, C., Using KADS methodology in a simulation assisted knowledge based system: application to hospital management. Expert Systems with Applications, 20(3), pp.235-249 (2001)

[32] Kafi, M.A., Challal, Y., Djenouri, D., Doudou, M., Bouabdallah, A. and Badache, N., A study of wireless sensor networks for urban traffic monitoring: applications and architectures. Procedia computer science, 19, pp.617-626 (2013).

[33] Bonino, D., Alizo, M.T.D., Alapetite, A., Gilbert, T., Axling, M., Udsen, H., Soto, J.A.C. and Spirito, M., August. Almanac: Internet of things for smart cities. In 2015 3rd International Conference on Future Internet of Things and Cloud (pp. 309-316). IEEE (2015).

[34] Mosser, S., Fleurey, F., Morin, B., Chauvel, F., Solberg, A. and Goutier, I., September. Sensapp as a reference platform to support cloud experiments: From the internet of things to the internet of services. In 2012 14th International Symposium on Symbolic and Numeric Algorithms for Scientific Computing (pp. 400-406). IEEE (2012).

[35] Chakraborty, S., Bhowmick, S., Talaga, P. and Agrawal, D.P., October. Fog networks in healthcare application. In 2016 IEEE 13th International Conference on Mobile Ad Hoc and Sensor Systems (MASS) (pp. 386-387). IEEE (2016).

[36] Ruggeri, G. and Briante, O., August. A framework for iot and e-health systems integration based on the social internet of things paradigm. In 2017 international symposium on wireless communication systems (ISWCS) (pp. 426-431). IEEE (2017).

[37] Hussain, M.I., Internet of Things: challenges and research opportunities. CSI transactions on ICT, 5(1), pp.87-95 (2017). 